\title{
Monoterpene isolated from the essential oil of Trachyspermum ammi is cytotoxic to multidrug-resistant Pseudomonas aeruginosa and Staphylococcus aureus strains
}

\author{
Faride Hosseinkhani ${ }^{[1]}$, Fereshteh Jabalameli ${ }^{[1]}$, Maryam Banar ${ }^{[1]}$, Nafiseh Abdellahi ${ }^{[1]}$, \\ Morovat Taherikalani ${ }^{[2]}$, Willem B. van Leeuwen ${ }^{[3]}$ and Mohammad Emaneini ${ }^{[1]}$
}

[1]. Department of Microbiology, School of Medicine, Tehran University of Medical Sciences, Tehran, Iran. [2]. Department of Microbiology, School of Medicine, Lorestan University of Medical Sciences, Khoramabad, Iran. [3]. Department of Innovative Molecular Diagnostics, University of Applied Sciences Leiden, Leiden, the Netherlands.

\begin{abstract}
Introduction: The aim of this study was to determine whether an herbal extract containing monoterpene exhibited activity against multidrug-resistant Staphylococcus aureus and Pseudomonas aeruginosa isolated from clinical infection samples. Methods: The essential oil of Trachyspermum ammi (L.) Sprague ex Turrill (Apiaceae) fruit was extracted by hydrodistillation. Fruit residues were treated with hydrochloric acid and re-hydrodistilled to obtain volatile compounds. Compounds in the distilled oil were identified using gas-chromatography (GC) and GC-mass spectrometry (MS). The antibiotic susceptibility of all bacterial isolates was analyzed using both the disc diffusion method and determination of the minimum inhibitory concentration (MIC). The sensitivity of antibiotic-resistant isolates to essential oil was also determined by using the disc diffusion method and MIC determination. Results: Of 26 clinical isolates, 92\% were multidrug-resistant (MDR). Aromatic monoterpenes (thymol, paracymene, and gamma-terpinene) were the major (90\%) components of the oil. Growth of S. aureus strains was successfully inhibited by the oil, with an inhibitory zone diameter (IZD) between $30-60 \mathrm{~mm}$ and $\mathrm{MIC}<0.02 \mu \mathrm{L} / \mathrm{mL}$. The oil had no antimicrobial activity against clinical isolates of $P$. aeruginosa; rather, it prevented pigment production in these isolates. Conclusions: This study revealed that the essential oil of Trachyspermum ammi, which contains monoterpene, has good antibacterial potency. Monoterpenes could thus be incorporated into antimicrobial ointment formulas in order to treat highly drug-resistant $S$. aureus infections. Our findings also underscore the utility of research on natural products in order to combat bacterial multidrug resistance.
\end{abstract}

Keywords: Thymol. Monoterpenes. Trachyspermum ammi. Staphylococcus aureus. Pseudomonas aeruginosa.

\section{INTRODUCTION}

Antibiotic resistance is a hallmark among many pathogens that pose serious public health risks ${ }^{(1)}$. Patients colonized or infected with multi drug resistant (MDR) bacteria including methicillin-resistant Staphylococcus aureus (MRSA) and Pseudomonas aeruginosa are more at risk of death than patients infected with non-resistant strains ${ }^{(2)(3)(4)}$. Treatment of such infections at the local level to prevent systemic infections is thus required. Hence, finding suitable alternatives to conventional antibiotic therapies are is a prerequisite in the battle against infectious diseases.

Herbal extracts are increasingly being considered as alternative antimicrobial agents. There are many advantages of using natural products as antimicrobial compounds,

\section{Corresponding author: Dr. Mohammad Emaneini.}

e-mail: emaneini@tums.ac.ir

Received 28 September 2015

Accepted 19 February 2016 including their generally low side effects, low costs, excellent biodegradability, and acceptance by patients due to their traditional applications and natural source ${ }^{(5)(6)}$. For thousands of years, Trachyspermum ammi (L.) Sprague ex Turrill (synonym: T. copticum) has been used as a medicinal plant in many parts of the world, ranging from Europe to Eastern Asia, and especially in Iran and India ${ }^{(7)}$. The brown seed-like fruit has traditionally been used for treatment of spasms, abdominal pains, and wound healing, but has also shown a positive effect on Helicobacter pylori infection ${ }^{(8)}$. Thymol, $\gamma$-terpinene, cymene, and limonene are the main components of the oil in this plant ${ }^{(9)(10)}$.

The aim of this study was to determine whether T. ammi essential oil exhibited cytotoxicity toward two clinically relevant pathogens, S. aureus and P. aeruginosa. Our data suggest that this oil could be used in formulations designed to treat wound infections.

\section{METHODS}

\section{Extraction}

The seed, Ajowan, was collected in a region of Iran (Yazd- Central Plateau of Iran) and the plants were grown 
in the Tehran University herbarium following institutional approval. Seeds were dried at room temperature. Oil distillation was performed as described previously ${ }^{(5)}$. Subsequently, $10 \mathrm{~mL}$ hydrochloric acid (Merck, Darmstadt, Germany) (1N) was added to the seed residue overnight at $25^{\circ} \mathrm{C}$ and hydrodistilled again for $4 \mathrm{~h}$. This acid treatment simplified the hydrolysis of glycosidic components.

\section{Gas chromatography analysis}

Gas chromatography (GC) of oil was performed using a Dani Master GC (Dani, Italy) with OV1 column (SE54CB, $25 \mathrm{~m} \times 0.25 \mathrm{~mm}$ i.d., $0.25 \mu \mathrm{m}$ film thickness), with nitrogen as carrier gas, a split ratio of 1:20, and a flame ionization detector (FID). Temperature programming was performed from $75^{\circ} \mathrm{C}$ (42 $\mathrm{min}$ ) to $250^{\circ} \mathrm{C}(14 \mathrm{~min})$ at a ramping of $15^{\circ} \mathrm{C} / \mathrm{min}$. Injector and detector temperatures were $250^{\circ} \mathrm{C}$ and $260^{\circ} \mathrm{C}$ respectively.

\section{Gas chromatography-mass spectrometry analysis}

The essential oils were also analyzed using the Gas chromatography-mass spectrometry (GC-MS) method on an Agilent 6890 with MS instrument (Agilent, U.S.) equipped with a BPX5 fused silica column $(30 \mathrm{~m} \times 0.25 \mathrm{~mm}$ i.d., film thickness $0.25 \mu \mathrm{m}$ ). The oven temperature was raised from $50^{\circ} \mathrm{C}$ through $300^{\circ} \mathrm{C}$ at a rate of $3^{\circ} \mathrm{C} / \mathrm{min}$ for $75 \mathrm{~min}$. The oven temperature was held for $5 \mathrm{~min}$ at $50^{\circ} \mathrm{C}$ and the transfer line temperature was $290^{\circ} \mathrm{C}$. Helium was used as a carrier gas at a flow rate of $0.8 \mathrm{~mL} / \mathrm{min}$ with a split ratio equal to $1 / 30$. The quadrupole mass spectrometer was scanned over an ionizing voltage of 70 electron volts $(\mathrm{eV})$ and an ionization current of $150 \mu \mathrm{A}$. The retention indices for all components were calculated using retention times of n-alkanes (C8-C25) injected after the essential oils under the same temperature and conditions. The compounds were identified by comparison of retention indices with those reported in the literature, together with comparison of their mass spectra with the Wiley, Adams, and National Institute of Standards and Technology (NIST) libraries.

\section{Bacterial strains}

Antibacterial efficacy of the oils was determined against 16 unique clinical isolates of $S$. aureus and 10 unique clinical isolates of P. aeruginosa, S. aureus ATCC 29213, and $P$. aeruginosa ATCC 27853. Clinical isolates were collected from burn wound infections in Tehran, Iran. The antibiotic susceptibility of all isolates (8 different classes for $S$. aureus isolates and 4 different classes for $P$. aeruginosa isolates) was analyzed using the disc diffusion method on Muller-Hinton agar (MHA) (Merck Co., Germany), as recommended by Clinical and Laboratory Standards Institute (CLSI) guidelines ${ }^{(11)}$. The tested antibiotics were purchased from Mast Group Ltd. (Merseyside, UK). Based on their resistance to antibiotics, clinical isolates were divided into different groups. Strains resistant to more than three different classes of antibiotic were defined as MDR. The study was approved by the Ethics Committee of Tehran University of Medical Sciences.

\section{Analysis of antimicrobial efficacy}

Disk diffusion assay: the disc agar diffusion (DAD) method was performed as described previously ${ }^{(13)}$. The bacterial suspension was adjusted to a density of $0.5 \mathrm{McFarland}$ to obtain an inoculum of approximately $1.0 \times 10^{8} \mathrm{CFU} / \mathrm{mL}$. A sterile swab immersed with this bacterial suspension was used to inoculate the entire MHA. Sterile filter paper discs (Whatman, $6 \mathrm{~mm}$ in diameter) were impregnated with $10 \mu \mathrm{L}$ of the oil and were placed on the inoculated agar plates (one disc per plate). Subsequently, agar plates were incubated for $24 \mathrm{~h}$ at $37^{\circ} \mathrm{C}$. The diameters of the inhibition zone were measured in millimeters and were compared with the results obtained from the reference strains $^{(12)(13)}$.

Minimum inhibitory concentration (MIC): the MIC was determined using the broth micro dilution method as recommended Clinical and Laboratory Standards Institute [CLSI (2013, M100-S23)]. The sample was subjected to a ten-fold dilution series in order to give final amounts of the original suspension ranging from $0.02-100 \mu \mathrm{L} /$ well. Thymol $\left(\mathrm{C}_{10} \mathrm{H}_{14} \mathrm{O}\right)$ was used as the positive control. All tests were performed in triplicate, and data are presented as mean \pm standard deviation (SD).

\section{RESULTS}

The results of the oil analysis by GC and GC-MS are shown in Table 1. Thirteen compounds were identified in the oil of the T. ammi before acid treatment, of which thymol (74\%), para-cymene $(16 \%)$, and $\gamma$-terpinene $(7 \%)$ were the major components. After acid treatment, thymol $(68 \%)$, para-cymene (14\%), and $\gamma$-terpinene (7\%) remained the major components.

The amount of aromatic monoterpenes in the oil before hydrolysis was higher than after acid treatment $(90 \%$ and $82 \%$, respectively). In contrast, after acid hydrolysis, the amount of monoterpene hydrocarbons and oxygenated monoterpene components was higher in the oil. The antibiotic susceptibility patterns and antibacterial efficacy of $T$. ammi are outlined in Table 2. The DAD method revealed that $T$. ammi essential oil was highly active against all $S$. aureus strains tested. There was no growth inhibition observed for the $P$. aeruginosa clinical isolates. The highest inhibition zone diameter (IZD) was achieved upon treatment of $S$. aureus ATCC 29213 and two clinical isolates (almost the whole plate was free of bacterial growth). In combination with the MIC results, the results from the disc diffusion method showed that all $S$. aureus strains tested were susceptible to the oil, and no growth was observed in any of the wells, with the exception of the negative control no extract well. None of the $P$. aeruginosa clinical strains were susceptible to the oil. Although reference strain P. aeruginosa ATCC 27853 had an average IZD before acid $(25 \mathrm{~mm})$ and after acid treatment $(10 \mathrm{~mm})$, it did not grow in any of wells and was thus classified as a susceptible strain. The highest MIC was measured in the P. aeruginosa standard isolate $(50 \mu \mathrm{L} / \mathrm{mL})$. To examine the major compounds within the oil, thymol was crystallized, and its antibacterial activity was tested at final concentrations of 1,10 , and $100 \mathrm{mg} / \mathrm{mL}$. Activity was only observed at the highest concentration $100 \mathrm{mg} / \mathrm{mL}$ of thymol, a dose that dramatically inhibited the growth of $S$. aureus strains. 
TABLE 1 - The most important chemical components of essential oils of Trachyspermum ammi before and after acid treatment.

\begin{tabular}{lccccc}
\hline & & & \multicolumn{2}{c}{ Percentage } \\
\cline { 3 - 5 } Number & Compound & $\mathbf{K I}^{\mathbf{1}}$ & $\mathbf{R T}^{\mathbf{2}}$ & $\mathbf{B A}^{\mathbf{3}}$ & $\mathbf{\mathbf { A A } ^ { \mathbf { 4 } }}$ \\
\hline 1 & thymol & 30.19 & 1,306 & 14 & 68 \\
2 & para-cymene & 16.63 & 1,031 & 7 & 14 \\
3 & gamma-terpinene & 18.32 & 1,063 & 0.5 & 7 \\
4 & carvacrol & 30.46 & 1,312 & 0.3 & 0.6 \\
5 & beta-pinene & 14.06 & 981 & 0.2 & 0.05 \\
6 & terpinen-4-ol & 24.71 & 1,190 & 0.2 & 0.1 \\
7 & carvone & 28.04 & 1,260 & 0.1 & 1.1 \\
8 & alpha-terpinene & 16.11 & 1,021 & - & 1.5 \\
9 & 1-terpineole & 22.43 & 1,144 & - & 1.1 \\
10 & $1,4-$ cineole & 16.02 & 1,019 & - & 0.5 \\
11 & alpha-terpinolene & 19.71 & 1,090 & - & 0.8 \\
12 & para-cymenene & 20.14 & 1,098 & - & 0.6 \\
13 & 1,8-cineole & 16.97 & 1,037 & & \\
\hline
\end{tabular}

1: kovats index; 2: retention time; 3: before acid treatment; 4: after acid treatment.

\section{DISCUSSION}

In this study, we investigated the antimicrobial efficacy of T. ammi essential oil against two relevant healthcare-associated bacteria, $S$. aureus and $P$. aeruginosa. Our data suggest that this oil could be suitable for the treatment of wound infections. Despite the variation in the proportion of minor oil compounds, the fraction of each of the major oil compounds before and after acid treatment was similar. Thymol was the most predominant component in oil both before and after acid treatment. With regard to the relative amounts of each essential oil component, our results are concordant with those of previous studies ${ }^{(14)(15)(16)(17)}$. The minor differences in results between several studies are due to the different extraction methods that were used. For example, some compounds identified in the oil after acid treatment, such as 1-terpineole, 1, 4 -cineole, paracymenene, and 1, 8-cineole, were absent prior to acid hydrolysis. Antibacterial effects of these minor compounds, an in particular 1, 4- cineole, have been previously reported ${ }^{(6)}$. Such compounds may be derived following acid hydrolysis of major hydrocarbons in the fruit cell walls ${ }^{(18)}$.

In general, the outer membrane of Gram-negative bacteria is less sensitive to the antimicrobial effects of essential oils ${ }^{(19)}$. The outer layer of the Gram-negative outer membrane is composed primarily of lipopolysaccharide molecules, and forms a hydrophilic permeability barrier that provides protection against highly hydrophobic drugs ${ }^{(6)}{ }^{(19)}$. This may explain the low sensitivity of Gram-negative bacteria to the cytotoxic effect of the lipophilic monoterpenes. In our study, essential oil did not exhibit antimicrobial activity against clinical isolates of $P$. aeruginosa.
However, the oil had a high bacteriostatic effect against all $S$. aureus clinical isolates, and elicited a relatively large IZD. For all $P$. aeruginosa strains, pigment production was inhibited near the disk. According to previous studies, the MIC values of plant oil against Gram-positive and Gram-negative bacteria ranged from 0.06 to $6 \mu \mathrm{L} / \mathrm{mL}^{(19)}$. In this survey, the MIC values for all S. aureus strains were consistently $<0.02 \mu \mathrm{L} / \mathrm{ml}$.

Thymol, a member of a natural class of phenolic monoterpene compounds known as biocides, was the predominant compound in T. ammi oil. Thymol has a strong antimicrobial effect either when used alone or in combination with other biocides such as carvacrol $^{(6)}{ }^{(20)}$. According to previous studies of Gram-positive test strains, these compounds strongly interfere with physiological characteristics such as cytoplasmic membrane permeability, coagulase activity, salt tolerance, and enterotoxin production ${ }^{(6)(21)}$. The oil may also influence the signal transduction in the $a g r$ system, a global regulator of virulence factor expression in staphylococci ${ }^{(21)}$. A previous study indicated that these types of mechanism of action were responsible for growth inhibition in $P$. aeruginosa reference strains ${ }^{(22)(23)}$. In contrast, our current data indicate that the oil and its derivatives were ineffective in highly resistance clinical isolates. The discrepancy in results may indicate that there are other molecular and cellular targets beyond outer membrane proteins that determine the sensitivity of Gram-negative bacteria, such $P$. aeruginosa, to monoterpene compounds. A previous study showed that monoterpene concentration determined the degree of resistance $P$. aeruginosa ${ }^{(22)}$. The MexAB-OprM efflux pump has an important role in the protection of $P$. aeruginosa against cyclic monoterpenes ${ }^{(24)}$. Considering that aromatic monoterpene compounds were the most predominant components both before 
TABLE 2 - Antibacterial activity of the essential oil of Trachyspermum ammi against clinical isolates of Staphylococcus aureus and Pseudomonas aeruginosa.

\begin{tabular}{|c|c|c|c|c|c|}
\hline Isolates & Number & $\begin{array}{c}\mathbf{B} A^{1} \\
\mathbf{I Z D}^{\mathbf{3}}(\mathrm{mm})\end{array}$ & $\begin{array}{c}\mathrm{AA}^{2} \\
\operatorname{IZD}(\mathbf{m m})\end{array}$ & $\mathrm{MIC} \mathrm{V/V}$ & Resistance pattern \\
\hline S. aureus ATCC 29253 & 1 & $\mathrm{WP}^{\dagger}$ & WP & $<0.02$ & \\
\hline \multirow[t]{10}{*}{ Clinical isolates of $S$. aureus } & 2 & 35 & 30 & ،, & E, GM, RP \\
\hline & 3 & 30 & 27 & ،, & $\mathrm{C}, \mathrm{GM}$ \\
\hline & 6 & 45 & 28 & ، & OX \\
\hline & 7 & 60 & 45 & ، & $\mathrm{CD}, \mathrm{E}, \mathrm{GM}, \mathrm{OX}, \mathrm{T}$ \\
\hline & 8 & 48 & 30 & ، & $\mathrm{E}, \mathrm{GM}, \mathrm{T}$ \\
\hline & 9 & 55 & 46 & “, & $\mathrm{E}, \mathrm{OX}, \mathrm{T}$ \\
\hline & 13 & 45 & 30 & ، & $\mathrm{CD}, \mathrm{E}, \mathrm{OX}, \mathrm{RP}$ \\
\hline & 14 & 40 & 28 & ،, & $\mathrm{CD}, \mathrm{E}, \mathrm{RP}$ \\
\hline & 15 & WP & WP & ،, & $\mathrm{CD}, \mathrm{E}, \mathrm{OX}$ \\
\hline & 16 & WP & WP & “' & $\mathrm{CD}, \mathrm{OX}, \mathrm{T}$ \\
\hline P. aeruginosa ATCC 27853 & 17 & 25 & 10 & 50 & \\
\hline \multirow[t]{5}{*}{ Clinical isolates of $P$. aeruginosa } & 18 & 0 & 0 & $>100$ & AK, CAZ, CPM, GM, IMI, MEM \\
\hline & 19 & 0 & 0 & ، & AK, CAZ, CPM, GM, IMI, MEM \\
\hline & 20 & 0 & 0 & ، & AK, CAZ, CPM, GM, IMI, MEM \\
\hline & 27 & 0 & 0 & ، & AK, CAZ, CPM, GM, IMI, MEM \\
\hline & 28 & 0 & 0 & ، & AK, CAZ, CPM, GM, IMI, MEM \\
\hline
\end{tabular}

BA: before acid treatment; AA: after acid treatment; IZD: inhibition zone diameter; $\uparrow \mathbf{W P}$ : growth inhibition in the whole plate. AK: amikacin; MIC: minimum inhibitory concentration; V/V: volume/volume. C: chloramphenicol; CAZ: ceftazidime; CD: clindamycin; CIP: ciprofloxacin; CPM: cefpiramide; E: erythromycin; GM: gentamicin; IMI: imipenem; MEM: meropenem; MUO: mupirocin; OX: oxacillin; RP: rifampin; T: tetracycline.

and after acid treatment of the oil, we suggest that resistance of clinical isolates of $P$. aeruginosa is due to the export of these compounds via efflux pumps present in these strains. Although the principle active ingredient appears to be thymol, we infer that other minor components in oil may synergize or antagonize with this monoterpene in a context-dependent manner.

In conclusion, our study revealed the effect of natural plant products on antimicrobial activity and highlights their potential utility as components of new antibiotics. Multidrug resistance among bacteria is a worldwide burden, and further research in natural products is thus needed.

\section{CONFLICT OF INTEREST}

The authors declare that there is no conflict of interest.

\section{FINANCIAL SUPPORT}

This research has been supported by Tehran University of Medical Sciences and Health Services (study grant number 25762/93-02-30). 


\section{REFERENCES}

1. Centers for Disease Control and Prevention (CDC). Get Smart: Know When Antibiotics Work. available: http://www.cdc.gov/ getsmart/community/about/index.html

2. Locksley RM, Cohen ML, Quinn TC, Tompkins LS, Coyle MB, Kirihara JM, et al. Multiply Antibiotic-Resistant Staphylococcus aureus: Introduction, Transmission, and Evolution of Nosocomial Infection. Ann Intern Med 1982; 97:317-324.

3. Kee EF, Robinson BJ, Hospenthal DR, Aldous WK, Wolf SE, Chung KK, et al. Prevalence of multidrug-resistant organisms recovered at a military burn center, Burns 2010; 36:819-825.

4. Kalantar Neyestanaki D, Mirsalehian A, Rezagholizadeh F, Jabalameli F, Taherikalani M, Emaneini M. Determination of extended spectrum beta-lactamases, metallo-beta-lactamases and AmpC-beta-lactamases among carbapenem resistant Pseudomonas aeruginosa isolated from burn patients, Burns 2014; 40:1556-613.

5. Hassanshahian M, Bayat Z, Saeidi S, Shiri Y. Antimicrobial activity of Trachyspermum ammi essential oil against human bacterial. Int J Adv Biol and Bioml Res 2014; 2:18-24

6. Nostro A, Sudano Roccaro A, Bisignan G. Effects of oregano, carvacrol and thymol on Staphylococcus aureus and Staphylococcus epidermidis biofilms. JMM Jour 2007; 56:519-523.

7. Bairwa R, Sodha RS, Rajawat BS. Trachyspermum ammi. Phcog Rev 2012; 6:56-60.

8. Paul S, Dubey RC, Maheswari DK, Kang SC. Trachyspermum ammi (L.) fruit essential oil influencing on membrane permeability and surface characteristics in inhibiting food-borne pathogens. Food Control 2011; 22:725-731.

9. Mahboubi M, Kazempour N. Chemical composition and antimicrobial activity of Satureja hortensis and Trachyspermum copticum essential oil. Iran J Microbiol 2011; 3:194-200.

10. Moazeni M, Saharkhiz MJ, Hosseini AA. In vitro lethal effect of ajowan (Trachyspermum ammi L.) essential oil on hydatid cyst protoscoleces. Vet Parasitol 2012; 187:203-208.

11. Clinical and Laboratory Standards Institute. Performance Standards for Antimicrobial Susceptibility Testing, M100-S23 (2013), M02-A11 and M07-A9. CLSI; 2013. Wayne, PA.

12. Kelen M, Tepe B. Chemical composition, antioxidant and antimicrobial properties of the essential oils of three Salvia species from Turkish flora. Biores Technol 2008; 99:4096-4104.

13. Sfeir J, Lefrançois C, Baudoux D, Derbré S, Licznar P. In vitro Antibacterial Activity of Essential Oils against Streptococcus pyogenes. Evid Based Complement Alternat Med 2013; 2013:269161.

14. Gandomi H, Abbaszadeh S, JebelliJavan A, Sharifzadeh A. Chemical constituents, antimicrobial and antioxidative effects of Trachyspermum ammi essential oil. J Food Process Preserv 2014; 38:1690-1695. DOI: 10.1111/jfpp.12131.

15. Velazhahan R, Vijayanandraj S, Vijayasamundeeswari A, Paranidharan V, Samiyappan R, Iwamoto T. Detoxification of aflatoxins by seed extracts of the medicinal plant, Trachyspermum ammi (L.) Sprague ex Turrill - structural analysis and biological toxicity of degradation product of aflatoxin G1. Food Control 2010; 21: 719-725.

16. Park IK, Kim J, Lee SG, Shin SC. Nematicidal activity of plant essential oils and components from ajowan (Trachyspermum ammi), allspice (Pimenta dioica) and litsea (Litseacubeba) essential oils against pine wood nematode (Bursaphelenchus Xylophilus). J Nematol 2007; 39: 275-279.

17. Nickavar B, Adeli A, Nickavar A. TLC-bioautography and GC-MS analyses for detection and identification of antioxidant constituents of Trachyspermum copticum essential oil. Iran J Pharm Res 2014; 13:127-133.

18. Solomon BD, Barnes JR, Halvorsen KE. Grain and cellulosic ethanol: History, economics, and energy policy. Biomass and Bioenergy 2007; 31:416-425.

19. Trombetta D, Castelli F, Sarpietro MG, Venuti V, Cristani M, Daniele C, et al. Mechanisms of Antibacterial Action of Three Monoterpenes. Antimicrob. Agents Chemother 2005; 49:2474-2478.

20. Palaniappan K, Holley RA. Use of natural antimicrobials to increase antibiotic susceptibility of drug resistant bacteria. Int $\mathrm{J}$ Food Microbiol 2010; 140:164-168.

21. Souza E, Oliveira CEV, Stamford TLM, Conceição ML, Gomes Neto NJ. Influence of carvacrol and thymol on the physiological attributes, enterotoxin production and surface characteristics of Staphylococcus aureus strains isolated from foods. Braz. J Microbiol 2013; 44:29-35.

22. Soumya E, koraichi S, Latrache H, Ghizlane Z, Hind M, Adnane R. Carvacrol and thymol components inhibiting Pseudomonas aeruginosa adherence and biofilm formation. Afr J Microbiol Res 2011; 5:3229-3232.

23. Zarrini G, Delgosha ZB, Moghaddam KM, Shahverdi AR. Postantibacterial effect of thymol, Pharm Biol 2010; 48:633-636.

24. Papadopoulos CJ, Carson CF, Chang BC, Riley TV. Role of the MexAB-OprM Efflux Pump of Pseudomonas aeruginosa in Tolerance to Tea Tree (Melaleuca alternifolia) Oil and Its Monoterpene Components Terpinen-4-ol, 1,8-Cineole, and $\alpha$-Terpineol. Appl Environ Microbiol 2008; 74: 932-1935. 